\title{
Structural and Magnetic Characterization of NiMnSb Half-Heusler Alloy Prepared by Rapid Quenching
}

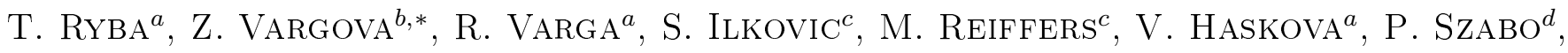 \\ J. KRAVCAK ${ }^{e}$, R. GYePES ${ }^{f}$ \\ ${ }^{a}$ Institute of Physics, Faculty of Sciences, P.J. Safarik University, Park Angelinum 9, 04154 Kosice, Slovakia \\ ${ }^{b}$ Dept. Inorg. Chem., Fac. Sci., UPJS, Moyzesova 11, 04154 Kosice, Slovakia \\ ${ }^{c}$ Univ. of Presov, Fac. Hum. and Nat. Sci., SK-08078 Presov, Slovakia \\ ${ }^{d}$ Centre of Low Temperature Physics, Institute of Experimental Physics SAS, Watsonova 47, 04001 Kosice \\ ${ }^{e}$ Dept. Phys., FEEI, Technical University of Kosice, Kosice, Slovakia \\ ${ }^{f}$ Dept. of Chemistry, Faculty of Education, J. Selye University, Komárno, Slovakia
}

\begin{abstract}
NiMnSb half-Heusler alloy in the form of a rod has been prepared by rapid quenching (suction-casting) method. The rod is characterized by single phase $\mathrm{C}_{b}$ crystalline structure with lattice parameter $a=5.982 \AA$. Magnetic measurements reveal an isotropic character of bulk NiMnSb alloy. Our study shows relatively high spin polarization of bulk NiMnSb in the range $35-50 \%$.
\end{abstract}

DOI: 10.12693 /APhysPolA.126.206

PACS: $75.30 . \mathrm{Gw}, 75.50 . \mathrm{Cc}$

\section{Introduction}

Heusler alloys are new perspective materials for many applications as spintronics, magnetocaloric cooling etc. The Heusler alloys are divided into two types: half Heusler alloys and full Heusler alloys [1-2]. Typical example of a half-Heusler alloy is NiMnSb. NiMnSb has general formula XYZ and crystallizes in noncentrosymmetric cubic structure $\mathrm{C}_{1 b}$. This composition is a very interesting material for application in spintronics devices due to its high spin polarisation, high Curie temperature and small value of Gilbert damping [3-4].

Typically, Heusler alloys are prepared by arc-melting method followed by high temperature (up to $1073 \mathrm{~K}$ [5]) and long time (up to 3 month [5]) annealing. In last years, the rapid quenching method has been applied for a fast production of large amount of alloys without the necessity of long thermal treatment [6].

In this contribution, we show the structural and magnetic characterization of NiMnSb half-Heusler alloy prepared by rapid quenching method. Alloy exhibits single crystalline phase with high Curie temperature and high isotropic magnetic properties. The local value of the spin polarization parameter in as-cast state ranges from 35 to $50 \%$.

\section{Experimental}

NiMnSb alloy was prepared by Arc-melting method followed by suction-casting into the Copper whole. This

*corresponding author; e-mail: vargovaz@upjs.sk method allows production of rods with $3 \mathrm{~mm}$ in diameter, having a length of $30 \mathrm{~mm}$.

The phase and microstructure were characterized by $\mathrm{X}$-ray diffraction (XRD, $\mathrm{Cu} \mathrm{K} \alpha$ ) and scanning electron microscopy (SEM) with EDX analyser.

The temperature dependence of the saturation magnetization and hysteresis loops for bulk were measured by vibrating sample magnetometer VERSALAB in Presov in the temperature range $10-400 \mathrm{~K}$ with applied magnetic field of $1 \mathrm{~T}$. The hysteresis loops were measured at $50 \mathrm{~K}$.

Point-Contact Andreev Reflexion Spectroscopy (PCAR) measurements through a microconstriction between a superconducting $\mathrm{Nb}$ tip and NiMnSb samples [7] have been used for the local study of spin polarization. In order to obtain the best spectral resolution, the experiments have been realized at temperatures (usually $T=1.5 \mathrm{~K}$ ) much lower than the superconducting transition temperature of $\mathrm{Nb}\left(T_{c}^{S}=9.2 \mathrm{~K}\right)$.

\section{Result and discussion}

SEM analysis of NiMnSb rod shows radial orientation of grains (see inset of Fig. 1) that results from high temperature gradient during suction casting. SEM/EDX confirms large homogenity of composition. Chemical composition, obtained from the EDX analyses, is similar to stoichiometric composition of NiMnSb, however there is a small lack of $\mathrm{Ni}$ and small excess of $\mathrm{Mn}$ $(\mathrm{Ni}=29.9 \%, \mathrm{Mn}=36.2 \%$ and $\mathrm{Sb}=33.9 \%)$.

XRD points to a single crystalline phase that was identified as crystalline $\mathrm{C}_{1 b}$ structure, space group $\mathrm{F} \overline{4} 3 \mathrm{~m}$ (see Fig. 1) with lattice parameters $a=5.982 \AA$.

Measurements of hysteresis loops in parallel and perpendicular direction with respect to rod axis (see inset of 
Fig. 2) confirmed relatively high isotropic structure (saturation fields in both directions are similar). However, the small anisotropy can be expected having easy magnetization axis in parallel direction. Hysteresis loops show coercive field $\mathrm{H}_{C} \sim 7.8$ Oe for parallel direction and 4.5 Oe for perpendicular direction. Estimated magnetic moment is $4.04 \mu_{\mathrm{B}}$ /f.u. that is comparable to the theoretically calculated one $\left(\sim 4 \mu_{\mathrm{B}} /\right.$ f.u. $)$. The Curie temperature exceeds our temperature range (400 K see Fig. 2). Typical $T_{\mathrm{C}}$ for NiMnSb should be in range of $730-1112 \mathrm{~K}$ [3].

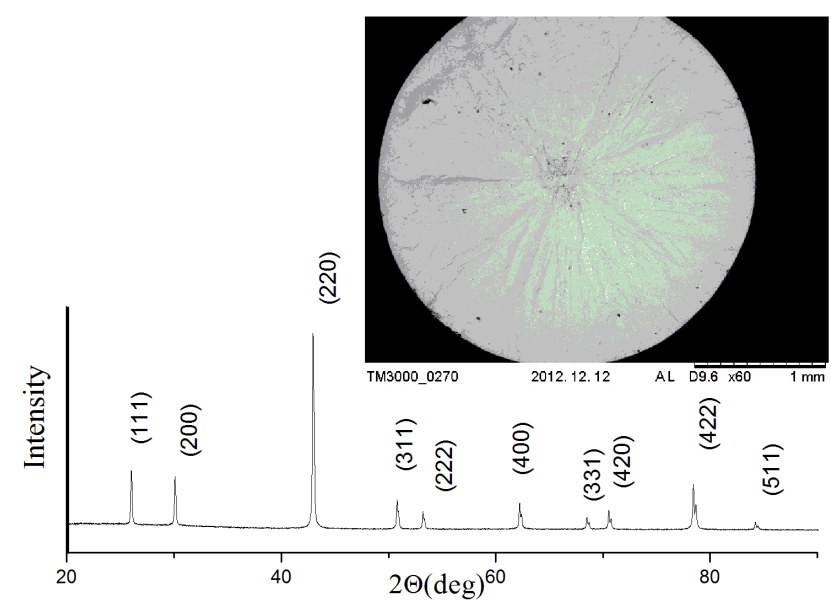

Fig. 1. RTG and SEM of NiMnSb in the form of bulk.

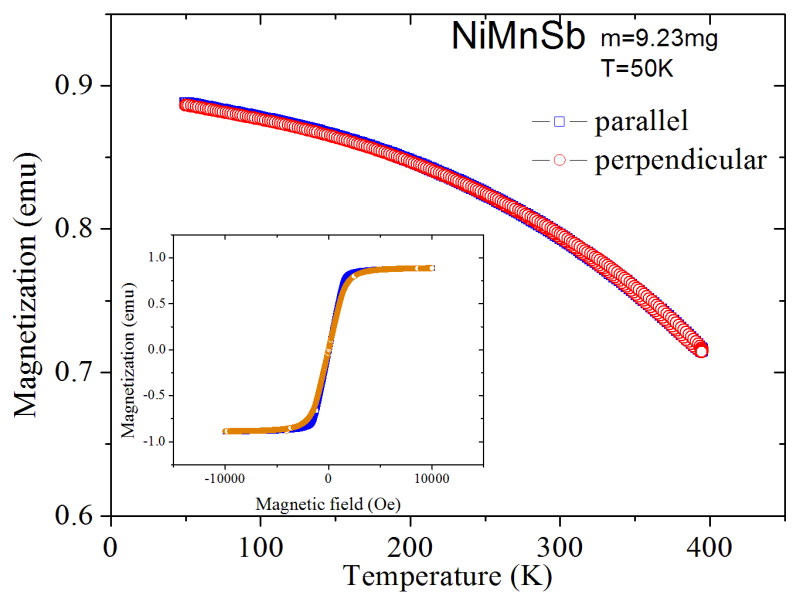

Fig. 2. Temperature dependence of magnetization of rapidly quenched $\mathrm{NiMnSb}$ alloy. Inset shows the hysteresis loops measured in parallel and perpendicular direction with respect to rod axis.

The spin polarization parameter $P$ has been determined from fitting of the PCAR spectra measured on different $\mathrm{Nb}-\mathrm{NiMnSb}$ point-contacts (at $T=1.5 \mathrm{~K}$ ) using the two-channel model of Strijkers et al. for spinpolarized PCAR currents $I_{P C}[7]$. In this model $I_{P C}$ is a weighted sum of a fully spin-polarized current $I_{s p}$ and a non-polarized Andreev reflexion $I_{A R}$. The weight $P$ of $I_{s p}$ defines the spin polarization parameter of the system. Via fitting our curves for this model we have obtained the local values of the spin polarization parameter $P$ scattered in the range $35-50 \%$, depending on the point-contact tip position. Typically, the measured spin polarizations for NiMnSb bulk alloys are in the range of $44-100 \%$ [3]. The lower value of spin polarization could be ascribed to small off-stoichiometric composition of our sample. Moreover, spin polarization is extremely sensitive to $\mathrm{X}-\mathrm{Y}$ and $\mathrm{X}-\mathrm{Z}$ disorder in XYZ half-Heusler alloy. Hence such disorder can also result in lower spin polarization of rapidly quenched Heuser alloy. Such a problem can be solved by re-annealing [6].

\section{Conclusions}

In the conclusion, we report on the fabrication of NiMnSb half-Heusler alloy by arc-melting followed by rapid quenching using suction casting method. Such a method allows production of rods having diameter of $3 \mathrm{~mm}$ and length of $30 \mathrm{~mm}$. Structural analysis shows single $\mathrm{C}_{1 b}$ structure (lattice parameters $a=5.982 \AA$ ) with the crystals gowth in radial direction (with respect to the rod axis).

Magnetic measurements reveal high isotropy of sample with a slight easy axis in axial direction. Magnetic moment is $4.04 \mu_{\mathrm{B}} / \mathrm{f}$.u. fits well the theoretically estimated values. The local value of spin polarization of rapidly quenched NiMnSb alloys varies within the range $35-50 \%$. The smaller value of spin polarization compared to another experiments could be ascribed to the slightly offstoichiometric composition or $\mathrm{X}-\mathrm{Y}$ and $\mathrm{X}-\mathrm{Z}$ disorder in $\mathrm{XYZ}$ alloy.

\section{Acknowledgments}

This work was supported by the project NanoCEXmat No. ITMS 26220120035, Slovak VEGA grant. No. $2 / 0135 / 13,1 / 0060 / 13$ and $2 / 0070 / 12$, APVV-0027-11 and APVV-0266-10

\section{References}

[1] T. Graf, S.S.P. Parkin, C. Felser, IEEE Trans. Magn. 47, 367 (2011).

[2] N.S. Bingham, H. Wang, F. Qin, H.X. Peng, J.F. Sun, V. Franco, H. Srikanth, M.H. Phan, Appl. Phys. Lett. 101, 102407 (2012).

[3] A. Hirohata, M. Kuchi, N. Tezuka, K. Inomata, J.S. Claydon, Y.B. Xu, G. van der Laan, Curent Opinion Solid State Materials Science 10, 93 (2006).

[4] D. Orgassa, H. Fujiwara,T.C. Schulthess, W.H. Butler, Phys. Rev. B 60, 13237 (1999).

[5] F. Wang, T. Fukuhara, K. Maezawa, K. Nishimura, F. Shimizu, S. Masubuchi, Jap. J. Appl. Phys. 49, 25502 (2010).

[6] J.L.S. Llamazeres, T. Sanchez, J.D. Santos, M.J. Perez, M.L. Sanchez, B. Hernando, L. Escoda, J.L. Sunol, R. Varga, Appl. Phys. Lett. 92, 012513 (2008).

[7] G. J. Strijkers, Y. Ji, F.Y. Yang, C.L. Chien, Phys. Rev. B 63, 104510 (2001). 